\title{
Avaliação das hemotransfusões alogênicas na maternidade do Hospital Universitário Regional do Norte do Paraná
}

\section{Evaluation of allogeneic hemotransfusions in the maternity ward of the Regional University Hospital of Northern Paraná}

\author{
Guilherme Gryschek $^{1}$; Adriane de Cássia Guergolet ${ }^{2}$; Kety Kimi Saito ${ }^{2}$; Vivian Uemura ${ }^{2}$; \\ Ameline Nishizima ${ }^{2}$; Francisco Pereira Silva ${ }^{3}$; Elbens M. M. Azevedo ${ }^{4}$
}

\section{Resumo}

\begin{abstract}
Os riscos inerentes às hemotransfusões alogênicas são amplamente conhecidos e, embora tenham diminuído nos últimos anos, parece improvável que possam ser completamente eliminados. É fundamental que se avalie corretamente cada paciente, para a adequada indicação transfusional ou a possível substituição por alternativas mais seguras, eficazes e acessíveis. No ciclo gravídico-puerperal, há maior exposição a riscos de anemia, quer a fisiológica quer a decorrente de hemorragias especialmente no periparto. Constituem, assim, um grupo de interesse para se considerar possíveis alternativas ao uso do sangue alogênico. O objetivo do presente estudo consiste nas principais indicações de transfusões na maternidade do Hospital Universitário Regional do Norte do Paraná (HURNP-UEL). Analisaram-se, retrospectivamente, 206 prontuários, de um total de 6552 pacientes internadas na Maternidade do HURNP, no período de maio de 1999 a julho de 2004, submetidas a 274 transfusões de sangue. Os principais dados coletados foram: epidemiológicos, nível de Hb pré-transfusional, indicações, tocurgias e complicações. A média etária foi de 27,5 (14-44) anos. As indicações mais freqüentes foram: anemia aguda (62\%), coagulopatia (7\%), anemia crônica (7\%), choque hipovolêmico (6\%) e outras (9\%). Em $9 \%$ dos casos, não se mencionou nenhuma indicação, e, neles, o nível médio de Hb pré-transfusional foi de $9,6 \mathrm{~g} / \mathrm{dl}$. Os produtos hemoterápicos mais utilizados foram: concentrado de hemácias $(82 \%)$, plaquetas (4\%), plasma (11\%) e crioprecipitado (3\%). O nível médio de $\mathrm{Hb}$ foi de $7,15 \mathrm{~g} / \mathrm{dl}$ nas pacientes que receberam concentrado de hemácias, dentre as quais em 5\% a $\mathrm{Hb}$ era maior ou igual a $10 \mathrm{~g} / \mathrm{dl}$. As tocurgias realizadas incluíram cesáreas, laqueaduras tubárias puerperais, histerectomias puerperais, curetagens, fórceps e partos normais com episiotomia. Conclui-se que a indicação de transfusão sangüínea não se deve basear apenas nos níveis de $\mathrm{Hb}$, mas em uma avaliação clínica criteriosa, cujos achados, muitas vezes, evidenciam que a mesma não é necessária. Na Maternidade do HURNP as hemotransfusões são realizadas dentro de critérios aceitáveis, mas é possível a sua diminuição no que concerne a um adequado enfoque terapêutico da anemia no ciclo grávido-puerperal,evitando-se, assim, as transfusões desnecessárias.
\end{abstract}

Palavras-chave: Transfusões alogênicas. Alternativas à hemotransfusão. Ciclo gravídico-puerperal.

\footnotetext{
${ }^{1}$ Terceiro ano de Medicina, Bolsista PIBIC/CNPq. E - mail: ggryschek@ gmail.com

${ }^{2}$ Terceiro ano de Medicina

${ }^{3}$ Professor doutor do Departamento de Clínica Cirúrgica/CCS da UEL.

${ }^{4}$ Professor Doutor Departamento de Ginecologia e Obstetrícia/CCS da UEL.
} 


\begin{abstract}
The risks inherent in allogeneic transfusions are well known. Although they have decreased in the last few years, it seems unlikely that they will be completely eliminated. It is essential that each patient is properly examined so that an appropriate transfusion is recommended or its possible substitution for safer, more effective and more accessible alternatives. During the gestational period there is more exposure to anemic risks, such as physiological anemia and puerperal bleedings. These women are thus considered a group of interest for possible alternatives for the allogeneic blood use. The purpose of this study was to evaluate the main transfusions recommendations in the Maternity Ward of the Regional University Hospital of Northern Paraná (HURNP - UEL). Two hundred and six patients' records were analyzed, out of a total of 6552 patients hospitalized in the Maternity Ward of HURNP, from May 1999 to July 2004, who had undergone 274 hemotransfusions. The main data gathered were: epidemiological information; $\mathrm{Hb}$ rates; transfusions recommendations; gynecological surgeries and complications. The average age was 27,5 (14-44) years old. The most common indications were: acute anemia (62\%), coagulopaties (7\%); chronic anemia (7\%), hypovolemic shock (6\%) and others (9\%). In $9 \%$ of the cases, no blood transfusions were mentioned. In these cases, the $\mathrm{Hb}$ average rate was $9.6 \mathrm{~g} / \mathrm{dl}$. The most used hemocomponents were: erythrocyte concentrate $(82 \%)$, platelets $(4 \%)$, plasma $(11 \%)$, and cryoprecipitate (3\%). The $\mathrm{Hb}$ average rate in the erythrocyte concentrate transfusion was $7,15 \mathrm{~g} / \mathrm{dl}$. In $5 \%$ of these cases, the hemocomponent was prescribed in rates equal to or greater than $10 \mathrm{~g} / \mathrm{dl}$. The obstetrical procedures included cesareans, tubal sterilizations, puerperal hysterectomies, uterine curettages, forceps and normal deliveries with or without episiotomies. It is concluded that the transfusions recommendations must not be based exclusively on $\mathrm{Hb} / \mathrm{Ht}$ rates. Although blood transfusions in the Maternity Ward of HURNP are carried out under acceptable criteria, it is possible to improve its recommendation concerning anemia during the pregnancy-puerperal cycle, avoiding unnecessary blood transfusions.
\end{abstract}

Key words: Allogeneic transfusions. Bloodless alternatives. Pregnancy and post-partum cycle.

\section{Introdução}

A segurança ao se empregar hemoderivados no tratamento de saúde tem preocupado a comunidade médica, bem como a população em geral, e isso deve conduzir à revisão do uso desses produtos, considerando que estão disponíveis alternativas efetivas e seguras, (MARTYN, 2002)

Os riscos do uso de produtos de bancos de sangue podem originar-se da incompatibilidade do sistema $\mathrm{ABO}$, além de reações relacionadas a outros anticorpos presentes no sangue doado. Complicações maiores, como a reação hemolítica fatal, podem ocorrer em 1: 600.000 unidades transfundidas, porém eventos não-letais, menos significativos, ocorrem com mais freqüência, e não são percebidos e notificados (KLEIN, 1995). Complicações infecciosas resultantes de sangue transfundido podem ser causadas por moléstias sexualmente transmissíveis, fato que tem assustado o público e servido de alarme ao problema de forma globalizada. Sistemas de Banco de Sangue do Canadá, França, Reino Unido e Japão têm sido afetados pelos olhos atentos do público cônscio da possibilidade de disseminação do sangue contaminado (PRESCOTT; SIMMONDS, 1998). A probabilidade de se obter produto alogênico completamente livre de patógenos é limitada em decorrência da própria natureza biológica de tais agentes. Podemos identificar e testar certo agente letal conhecido, mas agentes não testados ou desconhecidos podem resultar em novas doenças. 
Nos Estados Unidos, sistemas regionais de bancos de sangue, têm alertado a população sobre a probabilidade de exposição a sangue contaminado e outras possíveis reações decorrentes de transfusões alogênicas. Os produtos hemoterápicos têm se tornado mais seguros devido à realização de mais testes em busca de agentes contaminantes, o que, todavia, não tem amenizado a desconfiança do público, que exige melhor manejo do problema, no emprego de alternativas efetivas e seguras (HUSTON, 1997). Além desses fatores, a qualidade e segurança das transfusões em países em desenvolvimento são de constante preocupação, visto que $82 \%$ da população mundial vive nesses países. Infra-estrutura apropriada, pessoal treinado e recursos financeiros estão em falta, e não há política e nem serviço nacional de sangue eficiente. A doença e morte resultante de transfusões inseguras podem ter efeito devastador em comunidades de países carentes (DHINGRA et al., 2004; MBANYA; TAKAN; NDUMBE, 2003; MBANYA; BINAM; KAPTUEL, 2001; BINAM et al., 1998).

O amplo uso do sangue no mundo industrializado o tem colocado como recurso indispensável ao arsenal médico. No entanto, somente $5 \%$ da população norte-americana são doadores e tal índice tem declinado nos últimos anos (TIMBERLAKE, 1988). Em outros países, há dificuldade em se encontrar doadores voluntários cujo sangue esteja isento de contaminação (MBANYA; BINAM; KAPTUEL, 2001; MBANYA; TAKAN; NDUMBE, 2003). Em vista de tal situação, a busca de alternativas ao uso de sangue alogênico, tais como a tolerância do estado anêmico, a hemodiluição normovolêmica aguda intra-operatória, a recuperação intra-operatória de células, a intervenção rápida e cuidadosa em situações emergenciais, além de outras, podem ser opções práticas e aceitáveis.

No Brasil, em um recente levantamento do Ministério da Saúde em 1999, foram constatadas 1.931.951 hemotransfusões, ou seja, cerca de
161,7 transfusões a cada mil internações (ORGANIZAÇÃO PAN-AMERICANA DA SAÚDE, 2001).

Há, no ciclo gravídico-puerperal, maior exposição a riscos de anemia, quer fisiológica quer em decorrência de hemorragias, especialmente no peri-parto (COSLOVSKY; REZENDE, 1998). Hemorragias puerperais continuam como uma importante causa de morte materna em países desenvolvidos, mas especialmente nos países em desenvolvimento. Casos de hemorragia severa ocorrem em 6,7 a cada mil partos, de modo que o reconhecimento e tratamento imediato de tal complicação são essenciais para prevenir a morbimortalidade (MCLINTOCK, 2005; HIGGINS, 2003; WATERSTONE; BEWLEY; WOLFE, 2001). Esses casos constituem assim, um grupo de interesse para se considerar possíveis alternativas ao uso do sangue alogênico, algo que alguns pesquisadores têm conseguido fazer com sucesso (AZEVEDO et al., 2001; THOMAS, 1998).

\section{Objetivo Geral}

Realizar avaliação das hemotransfusões e critérios para sua realização em pacientes no ciclo grávido-puerperal internadas na Maternidade do Hospital Universitário Regional do Norte do Paraná (HURNP).

\section{Objetivos Específicos}

- Avaliar as indicações das hemotransfusões.

- Estudar as complicações decorrentes das hemotransfusões.

- Comparar resultados encontrados com os da literatura médica atual.

\section{Procedimentos Metodológicos}


Realizou-se uma busca em prontuários do HURNP e Ambulatórios do Hospital de Clínicas (AHC-UEL), da Universidade Estadual de Londrina, de dados de pacientes internadas na Maternidade do HURNP, no período de maio de 1999 até julho de 2004. Os dados foram colhidos por seis acadêmicos de Medicina segundo protocolo estabelecido (Anexo). Os prontuários dessas pacientes se constituíram única fonte das informações conseguidas.

Os dados então colhidos foram analisados no software Excel.

\section{Resultados}

No período citado, analisaram-se 206 pacientes que receberam algum tipo de hemocomponente, segundo dados fornecidos pelo banco de sangue do Hospital Universitário Regional do Norte do Paraná (HURNP). O número total de pacientes que receberam transfusão perfez 219 , e devido à falha no arquivo de prontuário, houve perda de 5,9\% da amostra, restando 206 pacientes. Considerou-se cada uma das indicações de transfusões como um evento separado, sendo analisados assim 274 procedimentos transfusionais nas supracitadas 206 pacientes. $\mathrm{O}$ total de pacientes internadas na Maternidade do HURNP no período foi de 6552; assim $3,15 \%$ das pacientes internadas receberam transfusão ou 41,8 procedimentos transfusionais a cada mil internações.

Observaram-se os dados epidemiológicos das pacientes, que constam de média etária: 27,5 anos (entre 14 e 44 anos); cor/raça; estado civil; e religião.

Em nenhum dos casos houve preocupação em se fazer um consentimento esclarecido às pacientes a respeito da terapêutica transfusional empregada.

As indicações mais freqüentes foram: anemia aguda (62\%), coagulopatia (7\%), anemia crônica (7\%), choque hipovolêmico (6\%) e outras (9\%) de caráter variado. Em 9\% dos casos não se mencionou nenhuma indicação por escrito, sendo o nível médio de $\mathrm{Hb}$ pré-transfusional de 9,6 g/dl.
As tocurgias realizadas foram variadas: 104 cesáreas, 13 laqueaduras tubárias, 5 histerectomias,

A freqüência dos hemocomponentes utilizados constou de: concentrado de hemácias $(82 \%)$, plaquetas (4\%), plasma (11\%) e crioprecipitado (3\%). A média do nível de $\mathrm{Hb}$ em que se realizou a infusão do concentrado de hemácias foi de 7,15 g/ dl. Em 5\% dos casos foi prescrita a transfusão de eritrócitos, em níveis maiores ou iguais a $10 \mathrm{~g} / \mathrm{dl}$. (Gráficos 3)

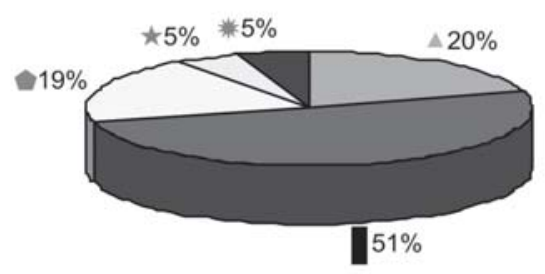

6 ou menos

$6-7,9 \wedge 8-9,9 \star 10$ ou mais $*$ * Não encontrado

Gráfico 3. Taxa de Hemoglobina pré-transfusional das pacientes que foram submetidas à hemotransfusões na Maternidade do HURNP

11 curetagens, 25 partos vaginais, sendo 15 normais com episiotomia e 3 com fórceps, e outros procedimentos cirúrgicos relacionados. (Gráfico 4).

Foram analisadas 100 metáfases por cultura, num total de 300 células por tratamento. Aberrações

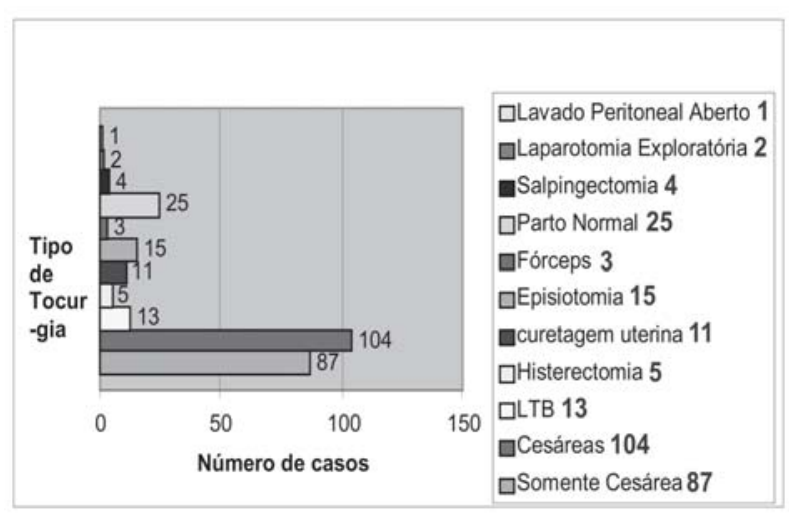

Gráfico 4. Tipos de Tocurgias em pacientes que foram submetidas à hemotransfusões na Maternidade do HURNP 
Como o hemocomponente mais utilizado foi o concentrado de hemácias, fez-se uma análise mais significativa do seu uso. Verificou-se sua utilização em pacientes que sofreram intervenção cirúrgica ou tocurgia, realizada em 150 pacientes. Assim, 160 episódios de hemotransfusão se relacionaram a algum tipo de tocurgia. Desses 160 episódios inicialmente relacionados com tocurgias, 99 se relacionaram com urgências e 31 com eletivas. Em 30 tocurgias não se encontrou o padrão de urgência ou eletiva.

Também se verificou o nível de $\mathrm{Hb}$ administrado nas transfusões de hemácias, cuja média para esse grupo foi de $6,9 \mathrm{~g} / \mathrm{dl}$. Ainda em 5\% dos casos instaurou-se a terapêutica com concentrado de hemácias embora o nível de $\mathrm{Hb}$ tenha sido superior a $10 \mathrm{~g} / \mathrm{dl}$.

Nas cirurgias eletivas, o nível médio de $\mathrm{Hb}$ prétransfusional foi $6,9 \mathrm{~g} / \mathrm{dl}$, embora em $31 \%$ dos eventos esse nível tenha sido superior a $8 \mathrm{~g} / \mathrm{dl}$ (embora abaixo de $10 \mathrm{~g} / \mathrm{dl}$ em todos os eventos). Ainda sobre tocurgias eletivas, a indicação mais freqüente foi anemia aguda, em 18 eventos, não sendo mencionada a indicação em outros 7 eventos.

Houve um caso em que se relatou ao banco de sangue uma reação transfusional febril, representando $0,36 \%$ de todos os 274 eventos analisados.

\section{Discussão}

A freqüência de hemotransfusões encontradas na Maternidade do HURNP de 3,15\%, com cerca de 41,8 procedimentos transfusionais a cada mil internações, se mostrou relativamente baixa, tendo em vista que no Brasil, em 1999, se realizaram 161,7 hemotransfusões em cada mil internações (ORGANIZAÇÃO PAN-AMERICANO DA SAÚDE, 2001). Não se encontraram dados específicos de transfusões em pacientes obstétricas de outros centros, e de modo algum se pode fazer equiparações quanto a gravidade das pacientes e a necessidade de hemotransfusões em seu tratamento, mas esse quesito pode servir de avaliação e comparação futura. Também a média geral do nível de hemoglobina pré-transfusional, de 7,15 g/dl, mostra que tem havido mais tolerância quanto ao estado anêmico das pacientes, que, geralmente se indica hemotransfusão quando atinge $8 \mathrm{~g} / \mathrm{dl}$. A clássica regra de 10 g/dl de $\mathrm{Hb}$ e $30 \%$ de Hematócrito, estabelecida nos anos 40 , foi usada como referência para se transfundir hemácias pela maioria dos médicos até a década de 80 . Sabia-se do perigo de pacientes com $7 \mathrm{~g} / \mathrm{dl}$ de $\mathrm{Hb}$ se submeterem a procedimentos cirúrgicos e que poucos sobreviviam à cirurgia com um nível inferior a 6 g/dl. Contudo, com mais descobertas sobre o carreamento do $\mathrm{O}_{2}$ e seu consumo em nível tecidual se questionou tal regra e desde 1988 se estabeleceu o parâmetro de $7 / 21$ ao invés do 10/30 (CONSENSUS CONFERENCE, 1988; GREENBURG, 1995).

No entanto, os dados mostram que algumas transfusões foram feitas sem necessidade aparente, visto trazerem como justificativa o nível de hemoglobina, da paciente e não seu estado clínico. A dúvida é se, frente aos dados obtidos, há falhas no preenchimento do prontuário, por não se deixar claro os motivos da indicação de hemotransfusão, ou na avaliação específica de cada paciente, tornando o procedimento desnecessário pelas dificuldades do profissional em avaliar a real necessidade das pacientes. Isso porque a indicação da transfusão eritrocitária não se deve basear apenas nos níveis sangüíneos de $\mathrm{Hb}$, mas, embasada em uma avaliação clínica criteriosa, cujos achados muitas vezes, evidenciam que a mesma não é necessária. Realmente, há muitos fatores que deveriam ser considerados na decisão de se fazer uma transfusão, incluindo volemia, duração e extensão da anemia e a presença de co-fatores fisiológicos que possam afetar a função cardiopulmonar e circulatória (GREENBURG, 1995). Nada indica nos dados verificados no presente estudo, em especial nos casos em que a transfusão se efetuou em níveis 
superiores a $10 \mathrm{~g} / \mathrm{dl}$ de $\mathrm{Hb}$, que tais fatores tenham sido levados em consideração.

Embora se trate de um procedimento de risco considerável associado a reações transfusionais e doenças transmissíveis, em nenhum dos casos houve preocupação em se fazer um consentimento esclarecido às pacientes. Esse tipo de declaração referente à vontade do paciente se faz cada vez mais importante, tanto para proteção do médico, como do paciente, que, desse modo, tem plena consciência do tipo de tratamento e de riscos a que está exposto.

O processo de aloimunização, desenvolvimento de anticorpos contra os antígenos associados com hemácias ou plaquetas transfundidas, é clinicamente silencioso, mas pode levar a numerosas complicações afetando futuras gestações e transfusões, sendo seu risco de $1 \%$ a cada unidade transfundida (GOLDBERG, 1995). Assim, não é possível identificá-lo como risco imediato, pois só será percebido em procedimentos e situações posteriores. Encontrou-se reação transfusional febril em um dos casos, representando $0,36 \%$ das transfusões. É um quadro benigno e bem comum, pois ocorre em $0,5 \%$ a $3 \%$ das transfusões em população de hospitais. Maior incidência é esperada em pacientes que recebem múltiplas transfusões (GOLDBERG, 1995).

O hemocentro do HURNP se mostrou bem eficaz quanto ao controle do uso do sangue estocado, visto não termos encontrado nenhum dado que sugira erros de compatibilidade sangüínea ou prazo de estocagem excedido. Entretanto, não foram avaliados outros tipos de complicações a longo prazo, tais como os diversos tipos conhecidos de vírus das hepatites e outros, além de questões imunológicas, inexoravelmente presentes (AZEVEDO et al., 2001). Embora os riscos tenham diminuído muito nas últimas décadas, se reconhece que persistem, mesmo se todas as providências disponíveis para evita-los forem adotadas.

Em cirurgias de urgência, a equipe médica tem menos tempo de planejamento e, portanto, menos controle sobre quaisquer imprevistos relacionados as condições clínicas do paciente ou as perdas sangüíneas intra-operatória. Mas, nas intervenções eletivas, e sujeitas ao planejamento cirúrgico e cuidadosa hemostasia intra-operatória, muitas transfusões de concentrados de hemácias talvez pudessem ter sido evitadas. Na presente casuística, das 31 cirurgias consideradas eletivas, a indicação em 18 delas foi referente a uma anemia aguda e em 7 não se esclareceu o motivo da transfusão. Como colocado anteriormente, as cirurgias eletivas são passíveis de planejamento ou cuidado intraoperatório (MARTYN et al., 2002).

Atualmente, existem muitas técnicas utilizáveis no peri-operatório, tais como o incremento da massa eritrócitária, adequada hemostasia, utilização de equipamentos que recuperam eritrócitos ("cell saver"), a hemodiluição normovolêmica aguda, fármacos que melhorem ou dificultem a diátese hemorrágica, para se evitar que o paciente alcance quadros anêmicos que necessitem hemotransfusão para sua correção (MARTYN et al., 2002). Em muitos centros médicos, as transfusões sangüíneas podem ser restringidas ao máximo, juntamente com seus riscos, por se dar preferência a um tratamento cuidadoso e bem planejado. Além disso, conhecer medidas alternativas para que, em casos específicos, possa evitar a exposição desnecessária do paciente a riscos é um dever do médico (WORD HEALTH ORGANIZATION, 1989).

\section{Conclusão}

Assim, considerou-se que, na maternidade do HURNP, as hemotransfusões são feitas dentro de critérios aceitáveis, como sendo nível de Hb prétransfusional abaixo de $8 \mathrm{~g} / \mathrm{dl}$ adequado à indicação do tratamento. Não há, entretanto, evidências de outros critérios que não o laboratorial. Desse modo, é de suma importância que se leve em consideração os aspectos clínicos das pacientes e se coloque a possibilidade de condutas alternativas, como reposição volêmica judiciosa, correção da anemia 
crônica, e tratamento da causa da hemorragia antes de se aventar a hemotransfusão. Se assim feito, evitar-se-iam, ao menos, os 5\% de transfusões de concentrado de hemácias feitas com níveis de $\mathrm{Hb}$ pré-transfusional igual ou acima de $10 \mathrm{~g} / \mathrm{dl}$. Ao se acatar medidas simples, é muito provável que a maioria dos casos em que se transfunde concentrado de hemácias não necessite de tal procedimento, beneficiando as pacientes, por isentálas dos riscos associados e o sistema de saúde, por conter os gastos relacionados com a medicina transfusional. Com isso, faz-se mister que os médicos busquem continuamente uma avaliação criteriosa e intervenção concorde, a fim de zelar pela saúde e bem-estar de suas pacientes.

\section{Agradecimentos}

Ao Professor Jefferson Rosa Cardoso, docente do Departamento de Fisioterapia do CCS-UEL , e ao Serviço de Arquivo (SAME) do AHC e HURNP da UEL, Londrina-PR

\section{Referências}

AZEVEDO, E. M. M.; AZEVEDO, M. F. V.; COUTO, J. O.; PASCOTINI, C. M. S. Alternativas ao uso de sangue alogênico em cirurgia ginecológica. Jornal Brasileiro de Medicina, Rio de Janeiro, v.80, n.1/2, p.92-96, 2001.

BINAM, F.; BOGNE, J. B.; TAKONGMI, S.; MELI, J. R.; KAPTUE, L. Transfusion practices and quantification of blood loss in a surgical setting in Cameroon. Medicina Tropical, Madrid, v.58, n.1, p.57-61, mar. 1998.

CONSENSUS CONFERENCE. Perioperative red blood cell transfusion. Journal of the American Medical Association, Chicago, v.260, n.18, p.2700-2703, 1988.
COSLOVSKY, S.; REZENDE, J. Repercussões da gravidez sobre o organismo. In: REZENDE, J. Obstetrícia. 8.ed. Rio de Janeiro: Guanabara Koogan, 1998. p.135-152

DHINGRA, N.; LLOYD, S. E.; FORDHAM, J.; AMIN, N. A. Challenges in global blood safety. World Hospitals and Health Services, London, v.40, n.1, p.45-49, 2004.

GOLDBERG, M. A. Erythropoiesis, erythropoetin, and iron metabolism in elective surgery: preoperative strategies for avoiding allogeneic blood exposure. American Journal of Surgery, New York, v.170, n.6A supl., p.37S-43S, 1995.

GREENBURG, A. G. A Physiology for red blood cell transfusion decisions. American Journal of Surgery, New York, v.170, n.6A supl., p.44S-48S, 1995.

HIGGINS, S. Obstetric haemorrage. Emergency Medicine, Princeton, v.15, n.3, p.227-231, 2003.

HUSTON, P. Building a blood system. In: BLOOD BANKS CONGRESSES, 21., 1997, Ottawa. Proceedings and Recommendations... Ottawa: Health Canada, 1997. p.59-88.

KLEIN, H. G. Allogeneic transfusion risks in the surgical patient. American Journal of Surgery, New York, v.170, n.6A supl., p.21S-26S, 1995.

MARTYN, V.; FARMER, S. L.; WREN, M. N.; TOWLER, S. C. B.; BETTA, J.; SHANDER, A.; SPENCE, R. K.; LEAHY, M. F. The theory and the practice of bloodless surgery. Transfusion and Apheresis Science, Kidllington, v.27, n.1, p.29-43, 2002.

MBANYA, D.; BINAM, F.; KAPTUE, L. Transfusion outcome in a resource-limited setting of Cameroon: a five-year evaluation. International Journal of Infectious Diseases, Brookline, Massachusetts, v.5, n.2, p.70-73, 2001. 
MBANYA, D.; TAKAN, D.; NDUMBE, P. M. Serological findingsamongst first-time blood donors in Yaoundé, Cameroon: is safe donation a reality or a myth?. Transfusion Medicine, Oxford, v.13, n.5, p.267-273, 2003.

MCLINTOCK, C. Postpartum haemorrhage. Thrombosis Research, Elmsford, v.115, n.1 supl., 2005.

ORGANIZAÇÃO PAN-AMERICANA DA SAÚDE. Perfil do sistema de serviços de saúde. Brasilia, 2001. Disponivel em: <http:// www.opas.org.br/servico/Arquivos/ perfil2000_wc.pdf>. Acesso em: 16 ago. 2006.

PRESCOTT, L. E.; SIMMONDS, P. Global distribution of transfusion transmited virus. The New England Journal of Medicine, New England, v.339, n.11, p.777-778, 1998.
THOMAS, J. M. The treatment of obstetric haemorrhage in women who refuse blood transfusion. British Journal of Obstetrics and Gynaecology, London, v.105, n.1, p.127-128, 1998.

TIMBERLAKE, G. A. Blood contaminated by enteric contents: an autotransfusion of potentially lifesaving measure in the massively hemorrhaging trauma patient? Journal of Trauma, Baltimore, v.28, n.6, p.855-857, 1988.

WATERSTONE, M.; BEWLEY, S.; WOLFE, C. Incidence and Predictors of Severe Obstetric Morbidity: Case-control Study. British Medical Journal, São Paulo, v.322, n.7294, p.1089-1094, 2001.

WORLD HEALTHORGANIZATION. Guidelines for the appropriate use of blood. Geneva: WHO, 1989. 


\section{Anexo}

Nome da paciente:

RGHU:

Data da internação:

Data da alta:

Evolução: ( ) Alta () Óbito

Local da internação: () HURNP () Maternidade Municipal

Data de nascimento: Cor/raça: ( ) branco ( ) pardo ( ) oriental ( ) negro

Naturalidade:

Estado Civil: () solteiro ( ) casado ( ) viúvo ( ) divorciado outro:

Religião:

Diagnóstico de admissão:

Tocurgia: ( ) sim () não

( ) eletiva ( ) urgência

Qual cirurgia?:

Tipo de anestesia: ( ) bloqueio ( ) geral

Indicação do Hemocomponente:
( ) Anemia aguda
( ) Coagulopatia
( ) Anemia crônica
( ) Desnutrição
( ) Choque hipovolêmico
( ) Outra:

Níveis séricos pré-transfusionais: $\mathrm{Hb}$ :

$\mathrm{Ht}:$

Plaquetas:

Consentimento informado para transfusão: ( ) sim () não

Tipo de Hemocomponente:
( ) Sangue total
( ) Crioprecipitado
( ) Concentrado de hemácias
( ) Plaquetas
() Plasma

Tempo de Estocagem:

Unidades utilizadas: ( ) 1 ( ) 2-3 ( ) 4 +

Complicações: () sim () não Qual?:

UTI: ( ) sim ( ) não

Tempo de Internação: 